\title{
Automatic radiometric normalization of multitemporal satellite imagery with the iteratively re-weighted MAD transformation
}

\author{
Morton J. Canty ${ }^{\mathrm{a}, *}$, Allan A. Nielsen ${ }^{\mathrm{b}, 1}$ \\ ${ }^{a}$ Institute for Chemistry and Dynamics of the Geosphere, Jülich Research Center, D-52425 Jülich, Germany \\ ${ }^{\mathrm{b}}$ Danish National Space Center, Technical University of Denmark, Richard Petersens Plads, Building 321, DK-2800 Kgs. Lyngby, Denmark
}

Received 27 March 2007; received in revised form 21 June 2007; accepted 15 July 2007

\begin{abstract}
A recently proposed method for automatic radiometric normalization of multi- and hyperspectral imagery based on the invariance property of the Multivariate Alteration Detection (MAD) transformation and orthogonal linear regression is extended by using an iterative re-weighting scheme involving no-change probabilities. The procedure is first investigated with partly artificial data and then applied to multitemporal, multispectral satellite imagery. Substantial improvement over the previous method is obtained for scenes which exhibit a high proportion of change.

(C) 2007 Elsevier Inc. All rights reserved.
\end{abstract}

Keywords: Change detection; Multispectral imagery; Iteratively reweighted multivariate alteration detection (IR-MAD); Canonical correlation analysis (CCA); Radiometric

\section{Introduction}

Ground reflectance determination from satellite imagery requires, among other things, an atmospheric correction algorithm and the associated atmospheric properties at the time of image acquisition. For most historical satellite scenes such data are not available and even for planned acquisitions they may be difficult to obtain. A relative normalization using the radiometric information intrinsic to the images themselves is an alternative whenever absolute surface reflectances are not required.

In performing relative radiometric normalization, one usually makes the assumption that the relationship between the at-sensor radiances recorded at two different times from regions of constant reflectance can be approximated by linear functions. The critical aspect is the determination of suitable time-invariant features upon which to base the normalization (Du et al., 2002; Furby and Campbell, 2001; Hall et al., 1991; Moran et al., 1992; Schott et al., 1988; Yang and Lo, 2000).

\footnotetext{
* Coresponding author. Tel.: +49 2461 614885; fax: +49 2461612496.

E-mail addresses: m.canty@fz-juelich.de (M.J. Canty), aa@space.dtu.dk (A.A. Nielsen).

${ }^{1}$ Tel.: +45 4525 3425; fax: +45 45881397.
}

In Canty et al. (2004) a fully automatic procedure was suggested for determining time-invariant observations which takes advantage of the invariance properties of the Multivariate Alteration Detection (MAD) transformation (Nielsen et al., 1998). The method was validated with unbiased statistical tests for equal means and variances of the normalized image bands and also compared quantitatively to manual selection of pseudo invariant features for normalization.

Since then, an iteratively re-weighted modification of the MAD transformation (IR-MAD) has been introduced (Nielsen, 2007) which establishes a better background of no change upon which to examine significant changes. The IR-MAD procedure is superior to the ordinary MAD transformation in identifying significant change, particularly for data sets in which the fraction of invariant pixels is relatively small, e.g., scenes which undergo large seasonal changes in vegetation or land use. It should therefore also perform better in isolating the no-change pixels suitable for use in relative radiometric normalization. In the present investigation this has been confirmed by comparing radiometric normalizations obtained with the original and with the iterated versions of the MAD transformation. Since the original MAD procedure has already been demonstrated to lead to as good or better normalizations than those achieved with manually determined invariant features (Canty et al., 2004; Schmidt et al., 


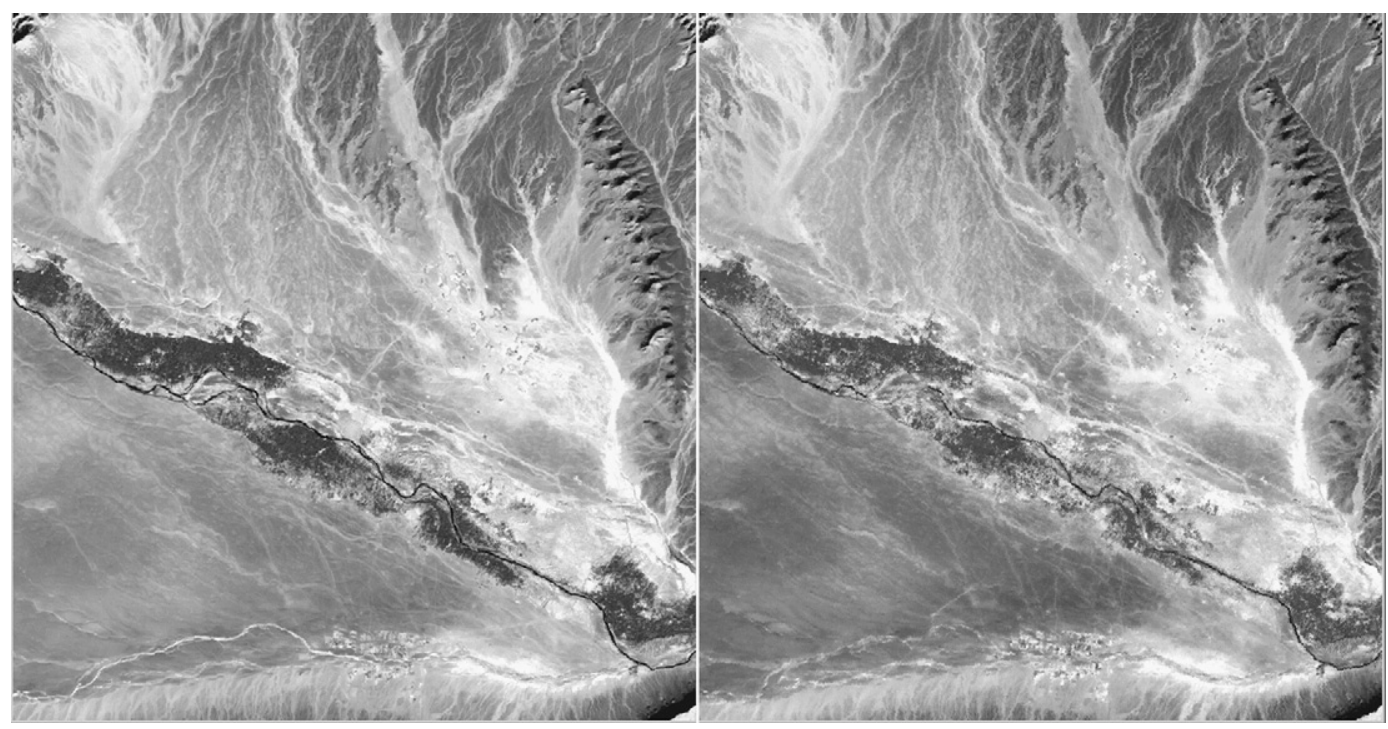

Fig. 1. Landsat ETM+ images over east central Morocco, left: December 19, 1999, right: October 18, 2000.

2005; Schroeder et al., 2006), further comparison with such methods is unnecessary.

Singh (1989) gives a good but now somewhat outdated survey of change detection algorithms for remotely sensed data. For recent reviews in a more general context see Radke et al. (2005) or Coppin et al. (2004). In Section 2 below we focus on the IR-MAD change detection procedure and briefly summarize its properties. The satellite imagery used for the comparison is described in Section 3. In Section 4 we apply the IR-MAD transformation to find invariant pixels for a partly artificial data set. In Section 5 results are described for a bitemporal arid scene in which relatively little change occurs, as well as for several image pairs exhibiting very substantial changes in ground reflectance. Invariant pixels obtained from IR-MAD are used to perform the necessary regressions for relative radiometric normalization and the quality of the normalized images is evaluated in terms of paired $t$-tests and $F$-tests for equal means and variances, respectively. Results are discussed and conclusions are drawn in Section 6.

\section{The MAD and IR-MAD transformations}

Consider first of all two $N$-band multispectral images of the same scene acquired at different times, between which acquisitions ground reflectance changes have occurred at some locations, but not everywhere. Assume without loss of generality both images to have pixel intensities with zero mean. Representing observations (pixel intensities) in the first multispectral image by a random vector $\boldsymbol{F}=\left(F_{1}, \ldots, F_{N}\right)^{\top}$, we can make a linear combination of the intensities for all spectral bands, creating a scalar image characterized by the random variable $U=\boldsymbol{a}^{\top} \boldsymbol{F}$. The vector of coefficients $\boldsymbol{a}$ is as yet unspecified. We do the same for the second image, represented by $\boldsymbol{G}$, forming the linear combination $\boldsymbol{V}=\boldsymbol{b}^{\top} \boldsymbol{G}$, and then look at the scalar difference image $U-V$. This combines

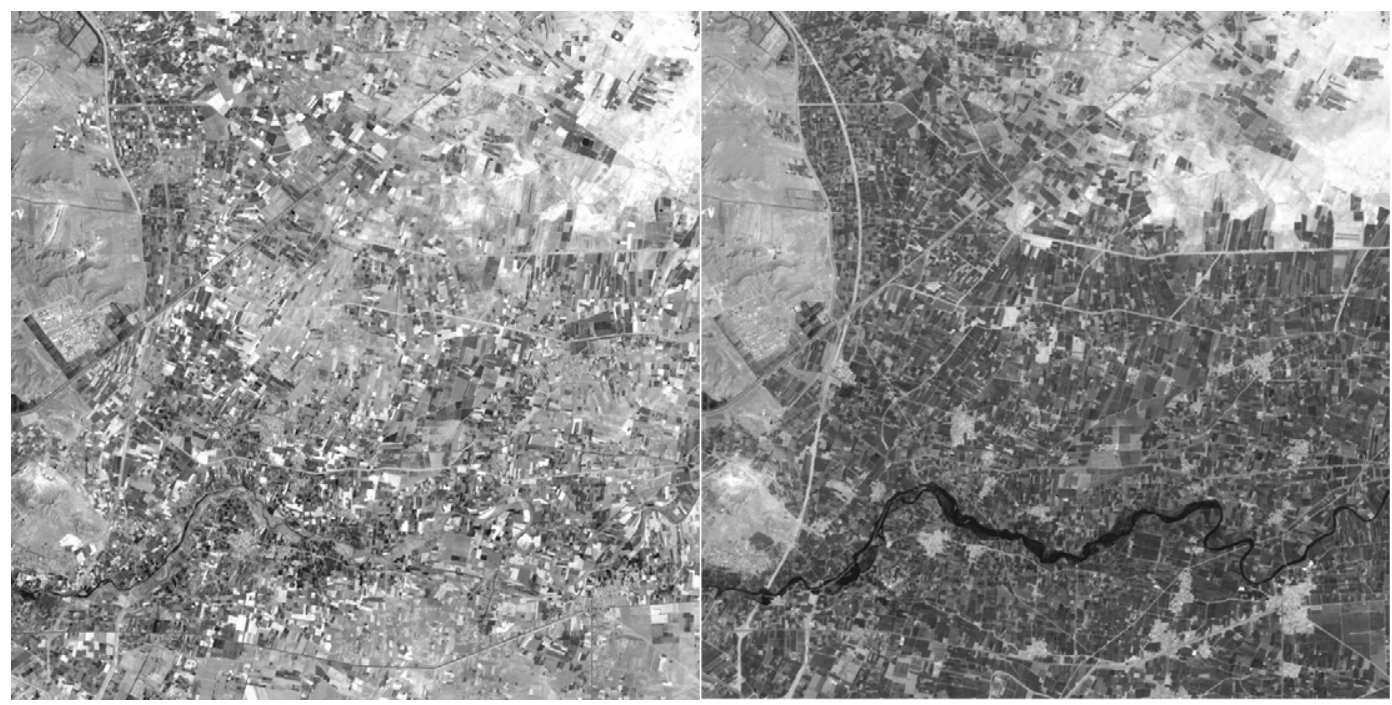

Fig. 2. ASTER images near Esfahan, Iran, left: July 30, 2001, right: May 22, 2005. 


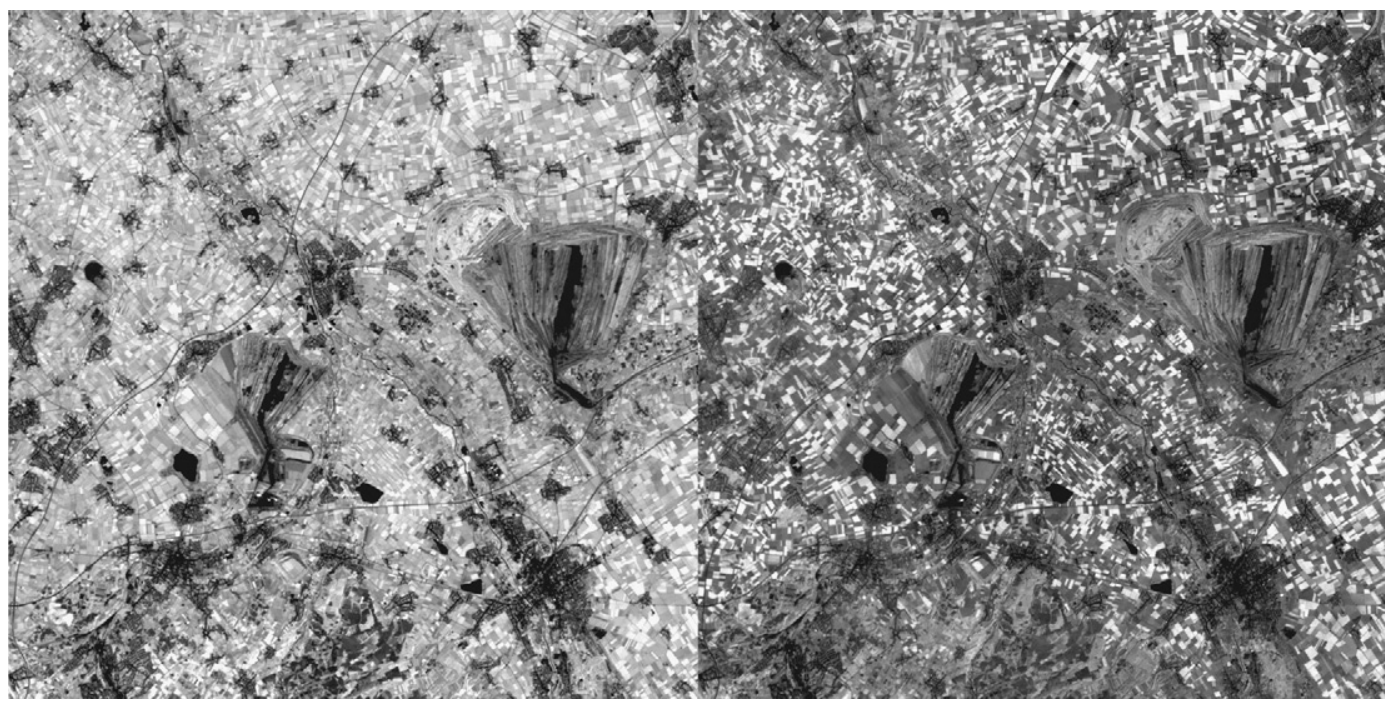

Fig. 3. Landsat ETM+ images over Jülich, Germany, left: June 26, 2001, right: August 29, 2001.

all of the change information into a single image, but one has of course still to choose the coefficients $\boldsymbol{a}$ and $\boldsymbol{b}$ in some suitable way. In Nielsen et al. (1998) it is suggested that they be determined by applying standard Canonical Correlation Analysis (CCA), first described by Hotelling (1936), to the images $\boldsymbol{F}$ and $\boldsymbol{G}$. This leads to the coupled generalized eigenvalue problems

$$
\begin{aligned}
& \boldsymbol{\Sigma}_{f g} \boldsymbol{\Sigma}_{g g}^{-1} \boldsymbol{\Sigma}_{g f} \mathbf{a}=\rho^{2} \boldsymbol{\Sigma}_{f f} \mathbf{a} \\
& \boldsymbol{\Sigma}_{g f} \boldsymbol{\Sigma}_{f f}^{-1} \boldsymbol{\Sigma}_{f g} \mathbf{b}=\rho^{2} \boldsymbol{\Sigma}_{g g} \mathbf{b},
\end{aligned}
$$

where $\boldsymbol{\Sigma}_{f f}$ and $\boldsymbol{\Sigma}_{g g}$ are the covariance matrices of the two images and $\boldsymbol{\Sigma}_{f g}=\boldsymbol{\Sigma}_{g f}^{\top}$ is the inter-image covariance matrix. Solution of the eigenvalue problems (Eq. (1)) generates new multispectral images $\boldsymbol{U}=\left(U_{1}, \ldots, U_{N}\right)^{\top}$ and $\boldsymbol{V}=\left(V_{1}, \ldots, V_{N}\right)^{\top}$, the components of which are called the canonical variates $(\mathrm{CVs})$. The CVs are ordered by similarity (correlation) rather than, as in the original images, by wavelength. The canonical correlations $\rho_{i}=\operatorname{corr}\left(U_{i}, V_{i}\right), i=1, \ldots, N$, are the square roots of the eigenvalues of the coupled eigenvalue problem and $\boldsymbol{a}_{\boldsymbol{i}}$ and $\boldsymbol{b}_{\boldsymbol{i}}, i=1, \ldots, N$, which determine $\boldsymbol{U}$ and $\boldsymbol{V}$ from $\boldsymbol{F}$ and $\boldsymbol{G}$, are the eigenvectors. The pair $U_{1}, V_{1}$ is maximally correlated, the pair
$\left(U_{2}, V_{2}\right)$ is maximally correlated subject to being orthogonal to (uncorrelated with) both $U_{1}$ and $V_{1}$, and so on. Performing paired differences (in reverse order) then generates a sequence of transformed difference images

$M_{i}=U_{N-i+1}-V_{N-i+1}, i=1 \ldots N$,

referred to as the MAD variates, having nice statistical properties which make them very useful for visualizing and analyzing change information. Thus for instance they are uncorrelated,

$\operatorname{cov}\left(M_{i}, M_{j}\right)=0, i \neq j$,

and have, by virtue of our chosen ordering, successively decreasing variances given by

$\operatorname{var}\left(M_{i}\right)=\sigma_{M_{i}}^{2}=2\left(1-\rho_{N-i+1}\right)$.

The fact that several additions and subtractions go into determining the MAD variates means that, from the Central Limit Theorem, they are ideally normally distributed. The usefulness of the MAD variates for automatic radiometric
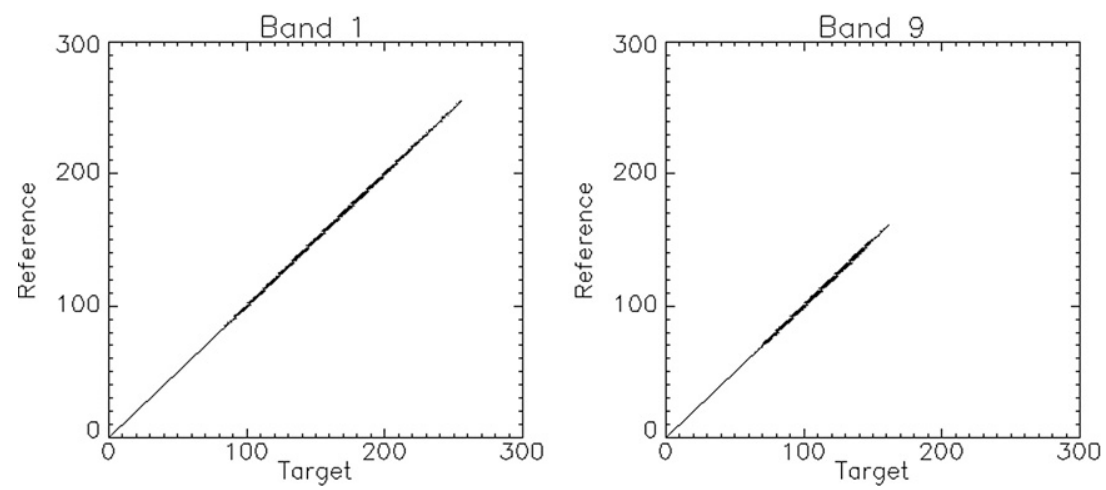

Fig. 4. IR-MAD normalization: Orthogonal regressions on spectral bands 1 and 9 for a $10 \%$ simulated no-change fraction in the ASTER bitemporal image. Only pixels identified as unchanged are plotted. 


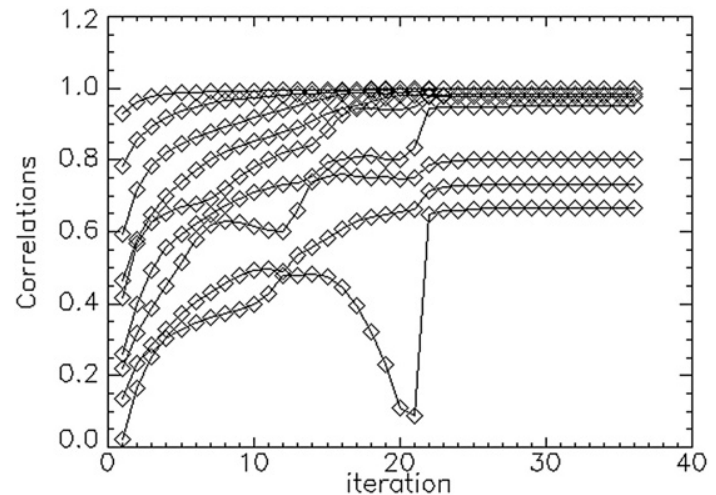

Fig. 5. Canonical correlations for the ASTER images with simulated no-change pixels.

normalization stems from the fact that they are invariant under affine transformations of either or both of the original images. This invariance is demonstrated explicitly in Canty et al. (2004).

Now consider two images of a scene, acquired at different times under similar illumination conditions, but for which no ground reflectance changes have occurred whatsoever. Then the only differences between them will be due to random effects like instrument noise and atmospheric fluctuation. In such a case we would expect that the histogram of any difference component that we generate would be very nearly Gaussian. In particular, the MAD variates, being uncorrelated, should follow a multivariate normal distribution with diagonal covariance matrix.

The MAD variates associated with change observations will deviate more or less strongly from such a multivariate normal distribution. Therefore, in the presence of genuine change, we expect an improvement of the sensitivity of the MAD transformation if we place emphasis on establishing an increasingly better background of no change against which to detect change. This can be done in an iteration scheme in which observations are weighted by the probability of no change, as determined on the preceding iteration, when estimating the sample means and covariance matrices which determine - via CCA - the MAD variates for the next iteration. ${ }^{2}$

The probability weights may be obtained by examining the MAD variates directly. Let the random variable $Z$ represent the sum of the squares of the standardized MAD variates:

$Z=\sum_{i-1}^{N}\left(\frac{M_{i}}{\sigma_{M_{i}}}\right)^{2}$,

where $\sigma_{M_{i}}$ is given by Eq. (4). Then, since the no-change observations are normally distributed and uncorrelated, their

\footnotetext{
${ }^{2}$ There is a close analogy here to the application of iterated principal component analysis (PCA) to bitemporal images for univariate change detection, see, e.g. Wiemker (1997). After one PCA transformation the first principal axis only corresponds approximately to the (highly correlated) nochange pixels, since it is determined by both no-change and change observations. Weighting the observations inversely to their distance from the first principal axis and repeating the transformation improves the position of the principal axes relative to the no-change pixels and, correspondingly, enhances the change signal.
}

realizations should be chi-square distributed with $N$ degrees of freedom (distribution function $P_{\chi^{2} ; N}(z)$ ). For each iteration, the observations $z$ (realizations of the random variable $Z$ ) can then be given weights determined by the chi-square distribution, namely

$\operatorname{Pr}($ no change $)=1-P_{\chi^{2} ; N}(z)$.

$\operatorname{Pr}$ (no change) is the probability that a sample $z$ drawn from the chi-square distribution could be that large or larger. A small $z$ implies a large probability of no change. Other weighting schemes are possible, for instance using unsupervised classification of change/no-change observations (Canty and Nielsen, 2006).

Iteration of the MAD transformation will continue until some stopping criterion is met, such as lack of change in the canonical correlations $\rho_{i}, i=1, \ldots, N$. In his original formulation of the iterative procedure, Nielsen (2007) standardized the MAD variates using the standard deviations of all of the observations, rather than using the iteratively re-weighted $\sigma_{M_{i}}$ values given by Eq. (4). We prefer here to use re-weighted values, as this was found to lead to considerably better performance for normalizing bitemporal scenes in which change dominates.

For radiometric normalization purposes, one can select all pixels which satisfy $\operatorname{Pr}$ (no change) $>t$ where $t$ is a decision threshold, typically $95 \%$. The preferred method for performing the actual normalizations is then to determine the necessary transformations from orthogonal - as opposed to ordinary linear regression on the invariant pixels, as demonstrated by Canty et al. (2004). A fraction of the invariant pixels may be withheld from the regression fit and used to test the normalized and reference images for equal radiometric characteristics.

In summary, the steps involved in radiometric normalization are as follows:

- Set weights equal to one for all pixels in the bitemporal scene.

- Repeat until canonical correlations cease to change significantly:

- Do a weighted sample the bitemporal image to determine its mean vector and covariance matrix.

- Perform CCA and construct the MAD variates $M_{i}, i=1, \ldots, N$.

- Recalculate the weights according to Eqs. (5) and (6).

Table 1

Comparison of means and variances for 7850 hold-out test pixels, with paired $t$-tests and $F$-tests for equal means and variances of the Morocco images, normalization using the original MAD transformation

\begin{tabular}{lcccccc}
\hline & Band 1 & Band 2 & Band 3 & Band 4 & Band 5 & Band 7 \\
\hline Target mean & 62.684 & 61.460 & 83.761 & 64.462 & 88.000 & 80.009 \\
Ref. mean & 75.509 & 68.501 & 91.131 & 73.193 & 98.775 & 90.335 \\
Norm. mean & 75.518 & 68.508 & 91.143 & 73.198 & 98.776 & 90.327 \\
$t$-stat. & -0.144 & -0.0688 & -0.0672 & -0.036 & -0.007 & 0.054 \\
$P$-value & 0.885 & 0.945 & 0.945 & 0.975 & 1.000 & 0.957 \\
Target var. & 10.61 & 28.83 & 87.78 & 55.46 & 95.24 & 59.63 \\
Ref. var. & 17.10 & 42.72 & 129.64 & 90.83 & 129.82 & 81.98 \\
Norm. var. & 17.54 & 43.34 & 131.16 & 91.70 & 131.80 & 83.42 \\
$F$-stat. & 1.025 & 1.014 & 1.011 & 1.009 & 1.015 & 1.017 \\
$P$-value & 0.255 & 0.524 & 0.606 & 0.672 & 0.504 & 0.440 \\
\hline
\end{tabular}


Table 2

As Table 1, but with 6671 hold-out test pixels and normalization after iteration of the MAD transformation to convergence

\begin{tabular}{|c|c|c|c|c|c|c|}
\hline & Band 1 & Band 2 & Band 3 & Band 4 & Band 5 & Band 7 \\
\hline Target mean & 62.617 & 61.269 & 83.632 & 64.305 & 87.021 & 79.314 \\
\hline Ref. mean & 75.535 & 68.498 & 91.339 & 73.186 & 98.097 & 89.940 \\
\hline Norm. mean & 75.543 & 68.479 & 91.321 & 73.174 & 98.073 & 89.915 \\
\hline$t$-stat. & -0.554 & 1.055 & 0.686 & 0.604 & 1.063 & 1.169 \\
\hline$P$-value & 0.580 & 0.292 & 0.493 & 0.546 & 0.288 & 0.243 \\
\hline Target var. & 9.98 & 26.36 & 79.34 & 50.22 & 93.64 & 58.14 \\
\hline Ref. var. & 14.94 & 37.70 & 116.46 & 79.96 & 115.39 & 70.65 \\
\hline Norm. var. & 15.25 & 38.11 & 116.71 & 80.38 & 115.09 & 70.31 \\
\hline$F$-stat. & 1.021 & 1.011 & 1.002 & 1.005 & 1.003 & 1.005 \\
\hline$P$-value & 0.392 & 0.654 & 0.931 & 0.828 & 0.916 & 0.844 \\
\hline
\end{tabular}

Table 3

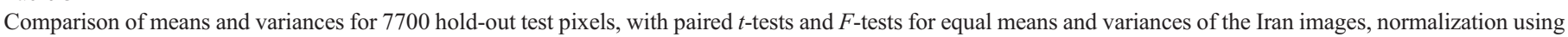
the original MAD transformation

\begin{tabular}{|c|c|c|c|c|c|c|c|c|c|}
\hline & Band 1 & Band 2 & Band $3 \mathrm{~N}$ & Band 4 & Band 5 & Band 6 & Band 7 & Band 8 & Band 9 \\
\hline $\begin{array}{l}\text { Target } \\
\text { mean }\end{array}$ & 83.105 & 95.208 & 129.564 & 119.392 & 111.495 & 118.465 & 109.393 & 105.607 & 101.404 \\
\hline Ref. mean & 190.244 & 177.438 & 127.689 & 129.593 & 129.235 & 136.017 & 123.814 & 124.005 & 130.920 \\
\hline $\begin{array}{l}\text { Norm. } \\
\text { mean }\end{array}$ & 190.206 & 177.382 & 127.707 & 129.591 & 129.230 & 136.001 & 123.805 & 124.005 & 130.899 \\
\hline$t$-stat. & 0.240 & 0.340 & -0.348 & 0.029 & 0.094 & 0.289 & 0.162 & 0.000 & 0.391 \\
\hline$P$-value & 0.810 & 0.734 & 0.728 & 0.977 & 0.926 & 0.772 & 0.871 & 1.000 & 0.696 \\
\hline Target var. & 179.39 & 349.25 & 153.34 & 326.10 & 333.29 & 407.37 & 339.39 & 365.58 & 268.70 \\
\hline Ref. var. & 587.00 & 607.97 & 166.91 & 98.32 & 90.87 & 113.23 & 97.09 & 146.29 & 96.90 \\
\hline $\begin{array}{c}\text { Norm. } \\
\text { var. }\end{array}$ & 704.39 & 680.72 & 168.75 & 82.62 & 80.89 & 99.69 & 83.88 & 123.50 & 85.86 \\
\hline$F$-stat. & 1.120 & 1.120 & 1.011 & 1.190 & 1.123 & 1.136 & 1.157 & 1.185 & 1.129 \\
\hline$P$-value & 0.000 & 0.000 & 0.000 & 0.000 & 0.000 & 0.000 & 0.000 & 0.000 & 0.000 \\
\hline
\end{tabular}

- Select pixels with no-change probability (Eq. (6)) exceeding a threshold $t$.

- Perform an orthogonal regression on the selected pixels to determine the relative radiometric normalization coefficients (slope and intercept).

\section{Imagery and software}

Three sets of satellite images were used for the study: a bitemporal LANDSAT ETM+ scene $(729 \times 754$ pixels $)$ over an arid area in Morocco (Fig. 1, the same data as were used to demonstrate the original normalization method (Canty et al., 2004)), multi-temporal $1000 \times 1000$-pixel ASTER scenes over an area southwest of Esfahan in Iran spanning a period of about four years (the set consisted of 5 image acquisitions in all, processed to "level $1 \mathrm{~b}$ - registered radiance at the sensor", two of which are shown in Fig. 2) and three LANDSAT ETM+ images $(1000 \times 1000$ pixels $)$ over the town of Jülich, Germany (Fig. 3 shows two of them). Extensive ground reference data were available for the Jülich acquisitions.

The image sets were registered to one another by applying an automatic contour matching algorithm (Li et al., 1995) and using first-order polynomial, nearest-neighbor resampling. The RMS errors were less than 0.5 pixel. In addition, the six short wave infrared (SWIR) bands of the ASTER images were sharpened to the $15 \mathrm{~m}$ resolution of the three visual near infrared (VNIR) bands

Table 4

As Table 3, but with 8400 hold-out test pixels and normalization after iteration of the MAD transformation to convergence

\begin{tabular}{lrrrrrrrrr}
\hline & \multicolumn{1}{c}{ Band 1 } & \multicolumn{1}{c}{ Band 2 } & Band 3N & Band 4 & Band 5 & Band 6 & Band 7 & Band 8 & Band 9 \\
\hline Target mean & 84.569 & 97.784 & 125.644 & 124.262 & 118.328 & 126.174 & 114.979 & 109.349 & 107.488 \\
Ref. mean & 188.163 & 175.520 & 124.158 & 130.804 & 132.247 & 139.710 & 125.520 & 123.571 & 134.177 \\
Norm. mean & 188.204 & 175.511 & 124.155 & 130.802 & 132.243 & 139.701 & 125.515 & 123.553 & 134.161 \\
$t$-stat. & -0.929 & 0.215 & 0.101 & 0.127 & 0.153 & 0.388 & 0.196 & 0.698 & 0.692 \\
$P$-value & 0.353 & 0.831 & 0.922 & 0.901 & 0.878 & 0.697 & 0.845 & 0.485 & 0.489 \\
Target var. & 50.067 & 80.689 & 101.680 & 52.979 & 30.015 & 40.225 & 30.887 & 68.753 & 19.037 \\
Ref. var. & 343.361 & 336.727 & 123.568 & 47.577 & 25.146 & 35.598 & 30.178 & 83.820 & 23.829 \\
Norm. var. & 352.881 & 342.440 & 122.949 & 46.742 & 24.620 & 35.138 & 29.591 & 83.054 & 24.157 \\
$F$-stat. & 1.028 & 1.017 & 1.005 & 1.018 & 1.021 & 1.013 & 1.020 & 1.009 \\
$P$-value & 0.210 & 0.440 & 0.818 & 0.416 & 0.332 & 0.551 & 0.367 & 0.673 & 0.532 \\
\hline
\end{tabular}



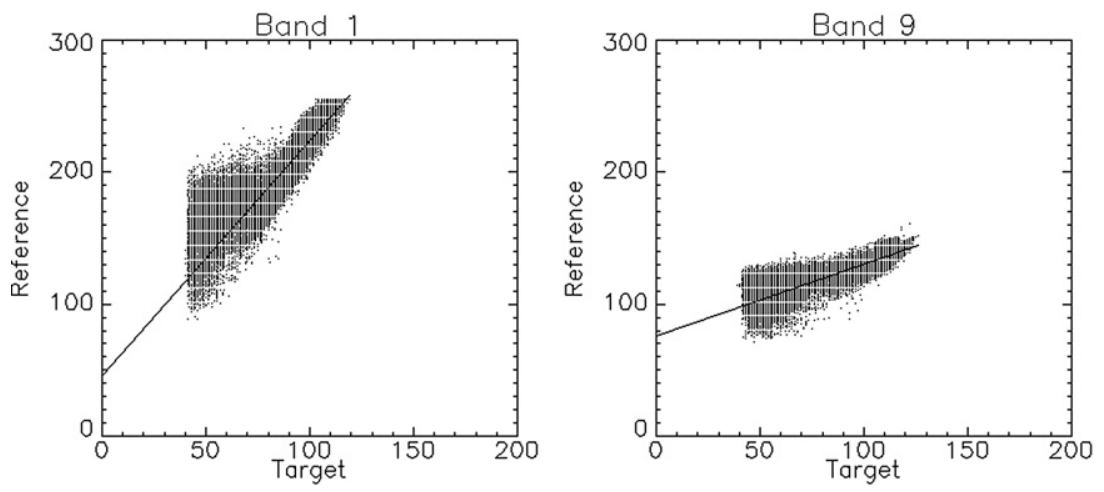

Fig. 6. Regressions on spectral bands 1 and 9 of the ASTER images for one iteration of the (i.e., the original) MAD transformation. Only pixels identified as unchanged are plotted.

with a wavelet fusion technique (Aiazzi et al., 2002). The ASTER images thus consisted of nine spectral bands each.

All image processing was performed within the ENVI remote sensing image analysis environment (ITT Visual Information Solutions). Extensions to ENVI for image registration, wavelet fusion, the IR-MAD transformation and radiometric normalization were written in the IDL language, see Canty (2007) for software availability.

\section{Simulated no-change observations}

To illustrate the effectiveness of IR-MAD in correctly identifying no-change observations, an artificial data set was generated from two of the ASTER images (those shown in Fig. 2) by copying a $316 \times 316 \times 9$ spatial/spectral subset from the upper left hand corner of the July 2001 image to the upper left hand corner of the May 2005 image, after adding a small amount of Gaussian noise (with standard deviation about $1 \%$ of the mean pixel intensity). The Gaussian noise prevents the solution of the coupled eigenvalue problem from becoming degenerate once the no-change pixels have been selected. The subset corresponds to a no-change fraction of $10 \%$ of the entire image.

The IR-MAD procedure identifies the copied pixels as no change to the exclusion of the rest of the scene. This is shown in Fig. 4, which displays the regression lines for radiometric normalization for spectral bands 1 and 9 . In the figure, reference refers to the image used as reference for the normalization and target is the image to be normalized. The regression lines go through the origin with $45^{\circ}$ slope and are determined entirely by the duplicated pixels. Fig. 5 shows the convergence of the canonical correlations for the artificial data set. The large changes in the correlations up to about the 20th iteration correspond to a "zeroing in" on the artificial no-change observations. Thereafter convergence is fast. When the size of the copied subset was reduced below $10 \%$, it was found that the iteration no longer converged to the duplicated pixels but rather to the physical no-change pixels. This is discussed in Section 6 .

\section{Real data}

We next investigated the effect of iterative re-weighting on radiometric normalization of a bitemporal arid scene, exemplified by the Morocco images, and of image pairs over Iran and Jülich which exhibited very significant seasonal and agricultural land-use changes.

\subsection{Morocco}

The statistics for the normalization of the October 2000 LANDSAT ETM+ image over Morocco (target) to the December
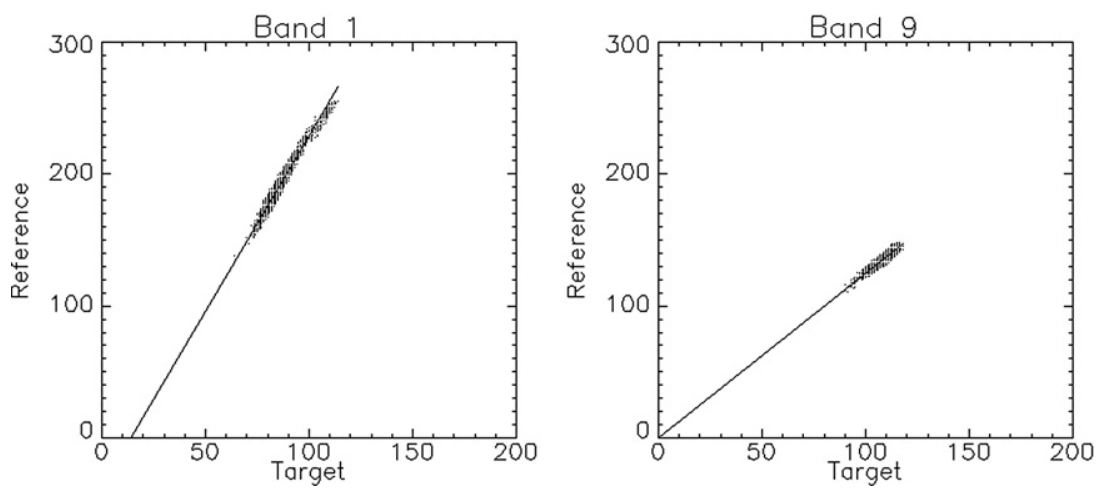

Fig. 7. Regressions on spectral bands 1 and 9 of the ASTER images after iteration of the MAD transformation to convergence. Only pixels identified as unchanged are plotted. 


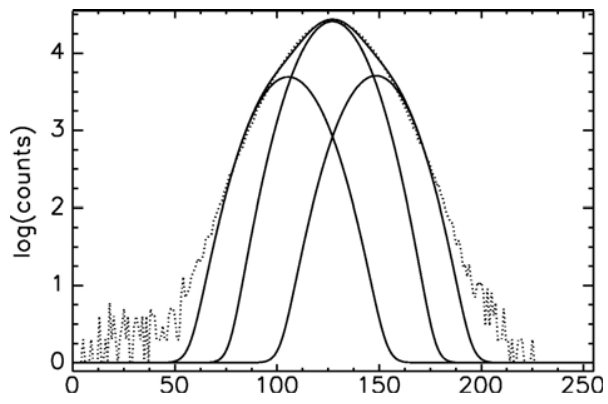

Fig. 8. Gaussian mixture model fit to the fifth MAD variate after one iteration.

1999 image (reference) using no-change pixels withheld from the normalization procedure are shown in Tables 1 and 2, the former for one iteration of the (i.e., the original) MAD transformation and the latter for iteration to convergence. Results for hypothesis tests for equal means and variances are included in terms of $P$-values. The $P$-values for tests for equal means and variances of the reference and normalized images are in both cases satisfactory, indicating that in this case a single iteration suffices for adequate identification of invariant pixels. (Given the observed value of a test statistic, the $P$ value is the lowest level of significance at which the null hypothesis could have been rejected, i.e., values close to one strongly support the null hypothesis of equal means and variances. Both the paired $t$-tests and the $F$-tests (for the hypotheses of equal means respectively variances after normalization) are so-called two-sided or two-tailed tests. In general we want paired $t$-test values close to zero (the closer the better) and $F$-test values close to one (the closer the better). In both cases we want $P$-values close to one (again, the closer the better). We accept the hypothesis of equal means or equal variances for $P$-values above some pre-set value which is traditionally often set to $5 \%$.)

\subsection{Iran}

In contrast to the Morocco scene, the ASTER images over Esfahan (see, e.g., Fig. 2) exhibit a very large proportion of change. The statistics for the radiometric normalization of the May 2005 ASTER image (target) to the July 2001 image (reference) using withheld no-change pixels are shown in

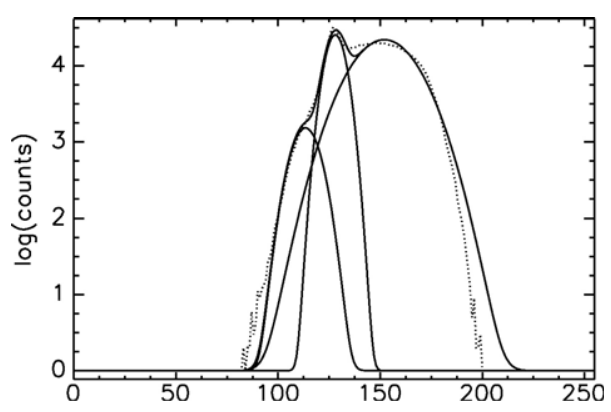

Fig. 9. Gaussian mixture model fit to the fifth MAD variate after iteration to convergence.

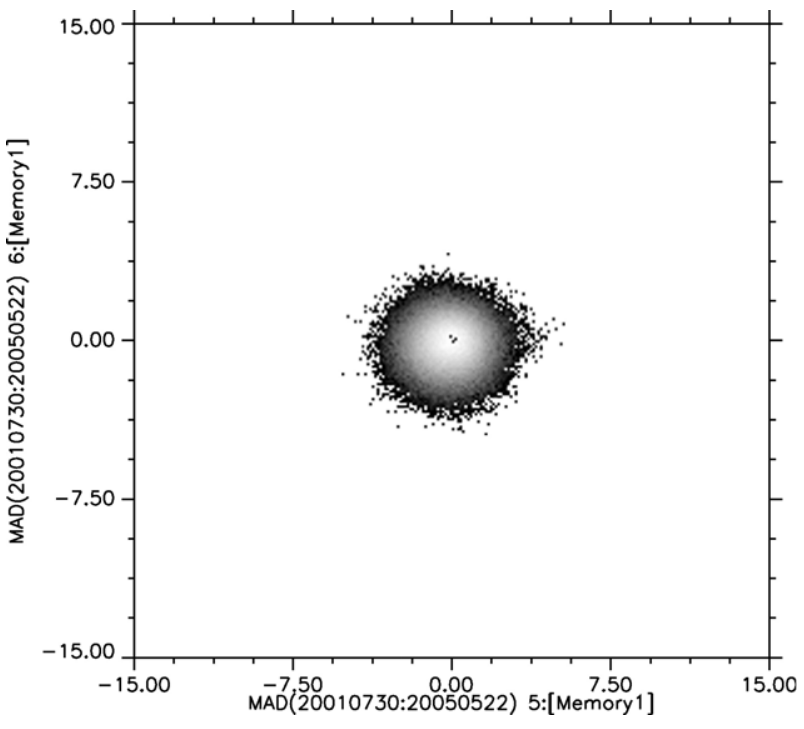

Fig. 10. Scatterplot of MAD variate 6 vs. MAD variate 5 for one iteration.

Tables 3 and 4, the former again for one iteration of the MAD transformation and the latter for iteration to convergence.

Again, the $P$-values for band-wise tests for equal means are acceptable in both cases, however the $P$-values for tests for equal variances of the reference and normalized images are satisfactory only for the IR-MAD result. Figs. 6 and 7 show typical regression lines obtained in the two cases. It is evident that invariant pixels are not properly discriminated after only one application of the MAD transformation, but that the iterative re-weighting scheme succeeds extremely well in isolating them.

This behavior is confirmed in Figs. $8-11$. Fig. 8 shows a 3-Gaussian mixture model fit on a logarithmic scale to one of the MAD variates after one iteration, where the central peak corresponds to no-change observations. The Gaussian density

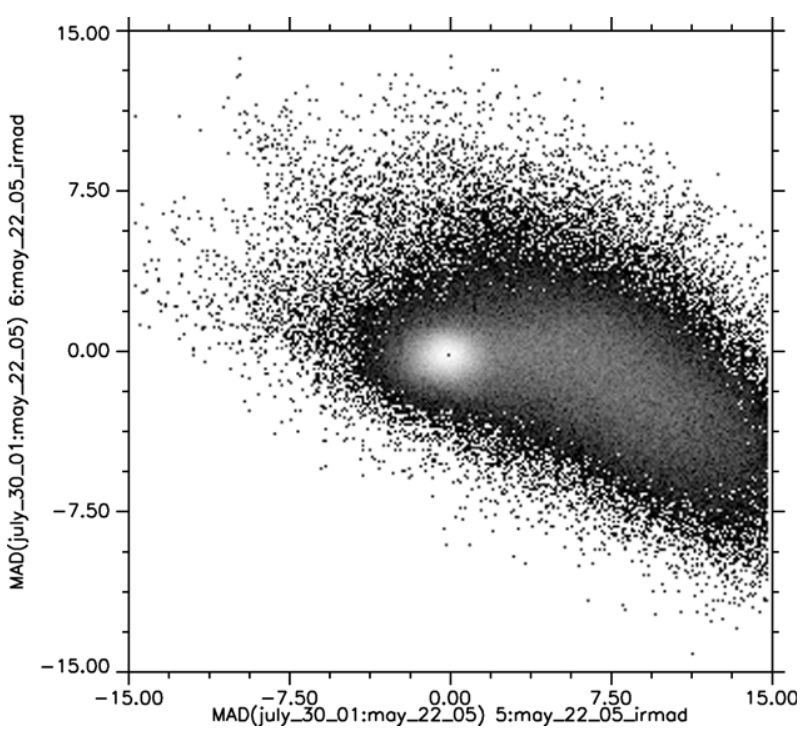

Fig. 11. As Fig. 10 after iteration to convergence. 


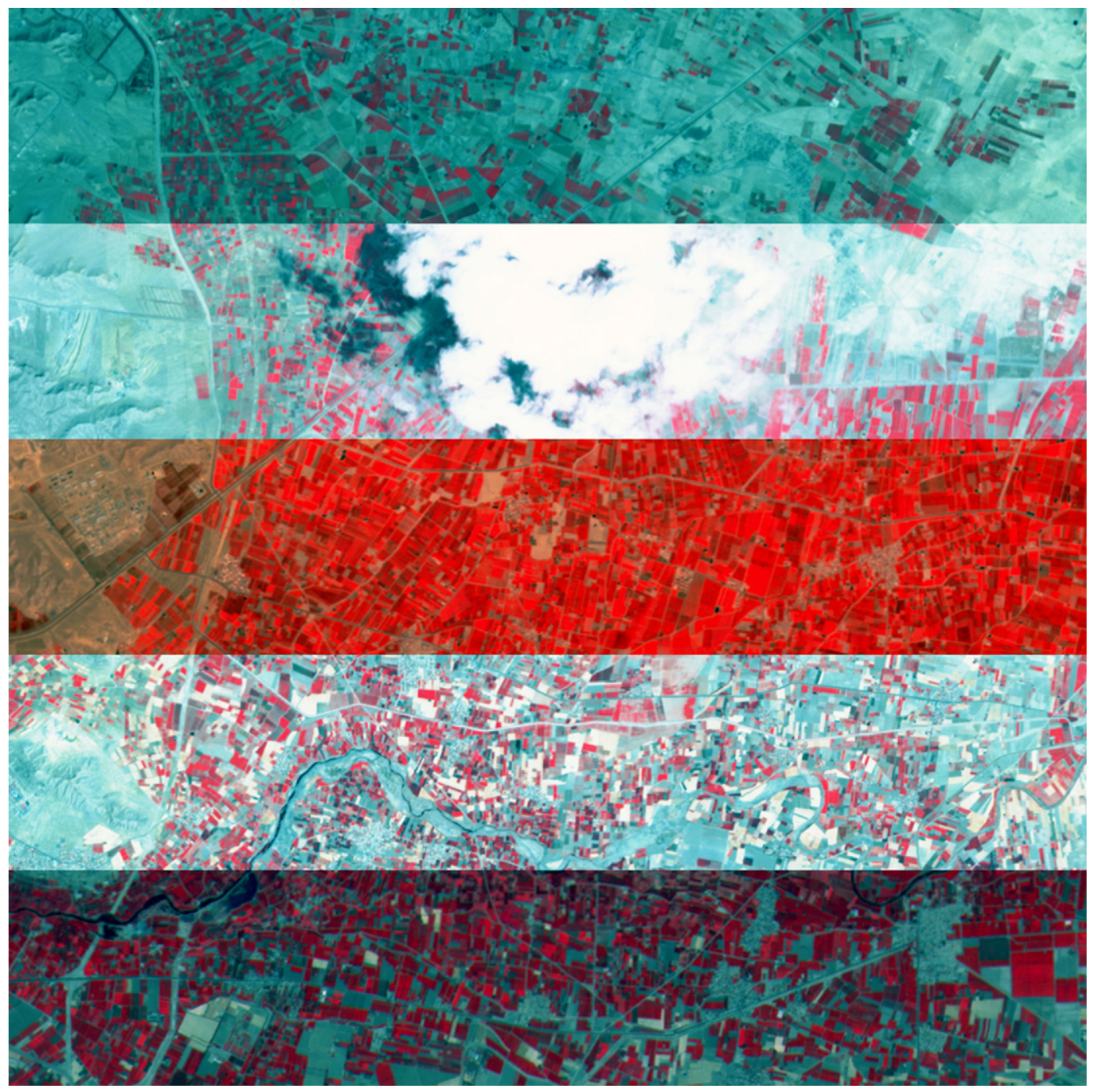

Fig. 12. Mosaic of 5 ASTER images over Iran prior to radiometric normalization, RGB color composite equalization stretch: band 1 (blue), band 2 (green), band 3N (red). The acquisition dates are, from top to bottom: March 11 2002, April 9 2001, May 22 2005, July 30, 2001 and September 112005.

parameters were estimated with the expectation maximization algorithm (Redner and Walker, 1984), see Canty and Nielsen (2006) for a detailed discussion. The same fit after iteration to convergence of the MAD transformation is shown in Fig. 9. There, a much better discrimination of the (relatively few) invariant pixels is clearly evident. The scatterplots in Figs. 10 and 11 indicate the same effect in the plane of MAD variates 5 and 6 . The no-change observations are the "hot spots" near the origin.

Horizontal mosaics of all 5 ASTER images before and after radiometric normalization to the July 302001 image are displayed in Figs. 12 and 13. The selected invariant pixels for each of the other four images, paired with the July 302001 scene, were used for the normalization. In Fig. 12 no gain corrections have been applied (this is unnecessary due to the linear invariance of the MAD transformation), partly accounting for the large intensity differences. For example, in the May 22, 2005 image, the 3N band gain was "normal" as opposed to "high" for the reference July 30 , 2001 image (see Abrams et al. (1999) for a definition of the
ASTER sensor gain categories). The remaining differences are due to varying atmospheric and solar illumination conditions as well as to true surface reflectance changes.

The success of the normalization is particularly evident on the left hand edge of Fig. 13, where a coherent arid area with no vegetation change can be followed from top to bottom of the mosaic with no discernible transitions, although very significant and real changes occur elsewhere in the scene. Note that the substantial cloud cover in the April 9, 2001 image has no effect on the quality of its normalization as all cloud and cloud shadow pixels are correctly identified as change.

\subsection{Germany}

The results of normalization of the May 25 and August 29, 2001 Jülich LANDSAT ETM+ images to the June 26, 2001 image were comparable to those for the ASTER images: A single application of the MAD transformation did not give acceptable $P$-values, whereas the IR-MAD method did. As an 


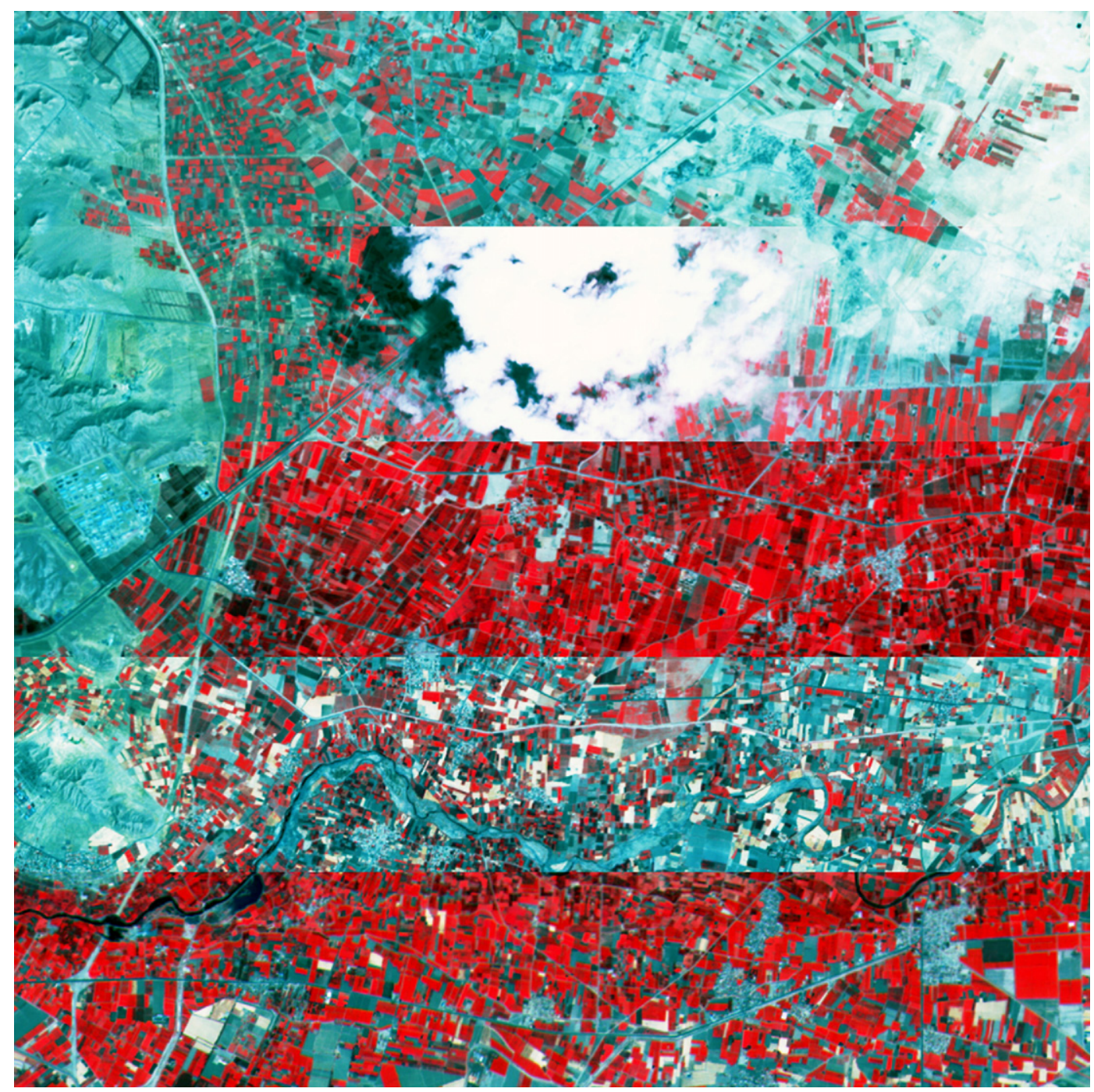

Fig. 13. As Fig. 12 after IR-MAD radiometric normalization.

example, Table 5 gives the comparison statistics for the August 29 (target) to June 26 (reference) normalization with the IRMAD procedure.

Fig. 14 displays a color composite of three IR-MAD variates for the Jülich scene showing changes between June 26 and August 29,

Table 5

Comparison of means and variances for 5850 hold-out test pixels, with paired $t$-tests and $t$-tests for equal means and variances of the Jülich images from June 26 and August 29 2001, normalization using the IR-MAD transformation

\begin{tabular}{lcccccc}
\hline & Band 1 & Band 2 & Band 3 & Band 4 & Band 5 & Band 7 \\
\hline Target mean & 67.388 & 51.217 & 45.538 & 64.086 & 60.583 & 37.262 \\
Ref. mean & 76.4787 & 58.517 & 49.235 & 85.411 & 72.198 & 41.288 \\
Norm. mean & 76.449 & 58.502 & 49.228 & 85.368 & 72.174 & 41.281 \\
$t$-stat. & 0.851 & 0.480 & 0.181 & 0.558 & 0.508 & 0.230 \\
$P$-value & 0.394 & 0.631 & 0.856 & 0.576 & 0.611 & 0.817 \\
Target var. & 62.08 & 79.80 & 165.64 & 262.50 & 206.82 & 178.03 \\
Ref. var. & 82.11 & 111.78 & 262.76 & 531.31 & 329.05 & 312.81 \\
Norm. var. & 81.92 & 111.96 & 263.92 & 542.57 & 331.76 & 316.15 \\
$F$-stat. & 1.002 & 1.001 & 1.004 & 1.021 & 1.008 & 1.010 \\
$P$-value & 0.931 & 0.951 & 0.866 & 0.422 & 0.753 & 0.6853 \\
\hline
\end{tabular}

2001. The intensities are stretched over \pm 8 standard deviations of the no-change pixels as determined from Gaussian mixture model fits like that shown in Fig. 9. (This very large dynamic range is indicative of the sensitivity of IR-MAD.) Middle gray indicates no change, while colored pixels signify change. Regions of prominent change are cultivated fields (principally due to maturing corn and sugar beet crops as well as grain harvesting), land reclamation in the wake of open pit mining, and mining activities themselves. The change categories are unrelated to one another and the MAD method associates them with different (orthogonal) MAD variates, as indicated by the coloring.

The invariant pixels found by setting a $95 \%$ threshold on the no-change probability (see Section 2) are indicated in yellow. They are concentrated in areas of urban settlement, small water bodies (flooded gravel pits) and mixed forest, where there is expected to be little or no change in canopy reflectance between June and August. There is, however, a conspicuous absence of no-change pixels in the "Eifel Forest" area at the bottom of the image, probably due to the more extreme bidirectional reflectance effects of local solar incidence angles associated 


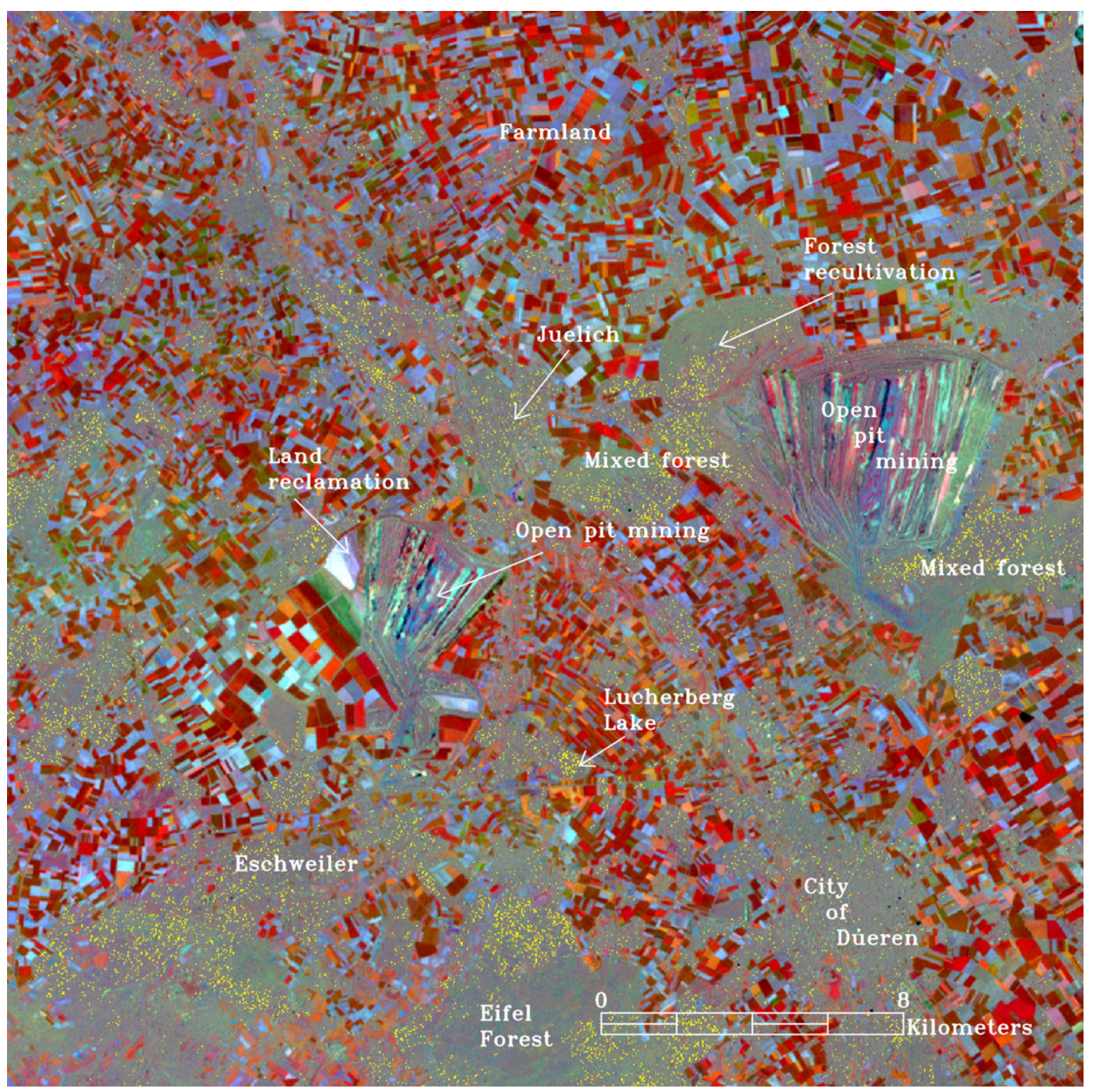

Fig. 14. RGB color composite of MAD variates 4 (blue), 5 (green) and 6 (red) for iteratively re-weighted MAD applied to the June 26 and August 29 Jülich images. The bands are stretched to \pm 8 standard deviations of the no-change pixels. Middle gray signifies no change. The 11,700 invariant pixels determined for radiometric normalization with the IR-MAD procedure are shown in yellow.

with the hilly topography of that region. If we define the nochange fraction to be the relative area under the central Gaussian of a three-Gaussian fit to an iterated MAD variate (see for example Fig. 9), then the no-change fraction for Fig. 14 is roughly $30 \%$. Only a small proportion of these (the yellow pixels) are used for normalization.

\section{Discussion and conclusions}

The ability of the iteratively reweighted MAD transformation to select invariant pixels in the presence of a high background of change has been demonstrated convincingly with partially simulated data. The statistical tests on holdout invariant pixels, as shown in Tables $1-5$, also indicate convincingly that, for real bitemporal scenes exhibiting a large amount of change, the MAD method for performing radiometric normalization is significantly improved by using iterative re-weighting.

As opposed to the change pixels, the invariant pixels determined by the MAD or IR-MAD methods appear to be spatially incoherent, see Fig. 14. This is due to the fact that a high threshold has been used to select them. The no-change observations on the whole will have, as we have indicated, a multivariate normal distribution. The decision threshold $t$ applied to the no-change probabilities, Eq. (6), will therefore have the effect of truncating most of the distribution and selecting only no-change observations with very small chi-square values. These will appear to be distributed randomly over the areas of no change.

Further insight into the IR-MAD method is provided in Tables 6-8, which illustrate the effect of iteration on the covariance structure of the MAD variates in the case of the Jülich data. Whereas, according to Eq. (3), after a single application of the MAD transformation the variance-covariance matrix of the MAD variates is necessarily diagonal (Table 6), after iteration this is no longer the case (Table 7). However, the variancecovariance matrix of MAD variates based on the weighted (i.e., the no-change) observations is diagonal, as shown in Table 8 . The diagonal values in Table 7 are much larger than the weighted 

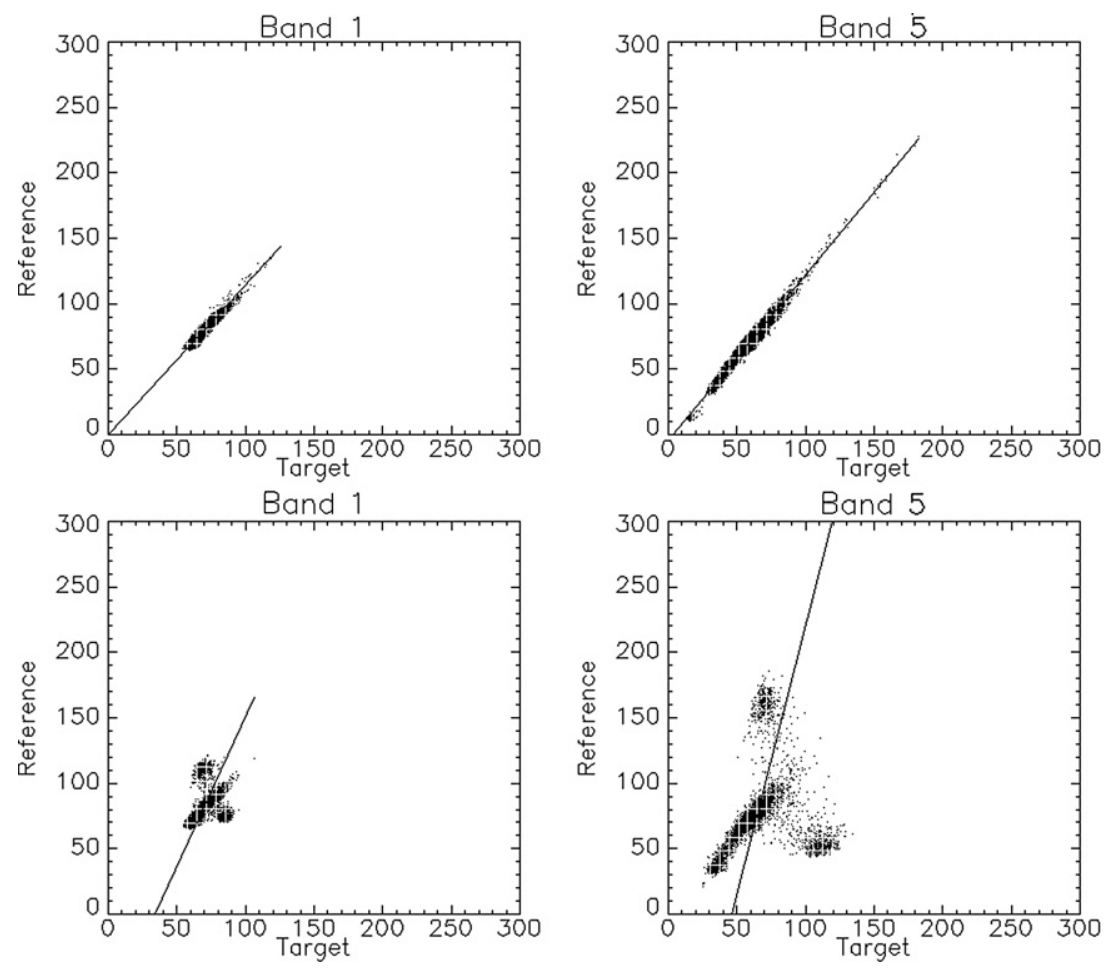

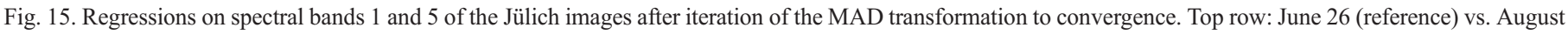
29 (target), bottom row: May 25 (reference) vs. August 29 (target). Only pixels identified as unchanged are plotted.

values of Table 8 and the off diagonal values are nonzero. They are in fact determined primarily by the change observations.

The normalization results for the May 2005 and July 2001 ASTER images discussed in Section 5.2 remain essentially unchanged if a 9-band spatial subset consisting of $<10 \%$ of the area of the first image is copied to the second image, whereas copying a larger subset will cause the IR-MAD algorithm to converge to the artificial no-change pixels, as was discussed in Section 4. The algorithm is evidently "attracted" to those pixels which are most strongly linearly correlated in the two acquisitions and which are in the majority, in the former case to the genuine no-change pixels at the expense of the artificial ones. It may be expected that there will be a lower limit on the number of truly invariant pixels in a bitemporal scene below which the IR-MAD algorithm will fail, in the sense that it will converge to noninvariant observations. This effect, which might be expected to be highly scene-dependent, is illustrated in Fig. 15 with the Jülich data. The regression lines for June 26 vs. August 29 (top row) are satisfactory and correspond to the results of Table 5. Those for May 25 vs. August 29 (bottom row) are obviously unsuitable for radiometric normalization. The seasonal differences between the two acquisitions (spring to late summer) are evidently too great. Placing a more severe threshold for identifying no-change observations did not remove the two "satellite" clusters in the scatterplots shown. On the other hand, normalization of the May 25 and August 29 images to the June 26 image posed no difficulty.

Quite generally we can conclude that, for multi-temporal images in which change is dominant, the IR-MAD transformation succeeds in identifying suitable invariant pixels for radiometric normalization where the single MAD transformation performs less successfully. For $1000 \times 1000 \times 6$-dimensional image arrays (e.g., the Jülich images), a single iteration of the MAD transformation, including determination of the weights for the next iteration, requires about 10 seconds CPU time on a 2.4 GHz Pentium 4 PC running IDL 6.2 under Windows XP. Convergence is usually satisfactory within $20-30$ iterations. For very large spatial/spectral subsets (for example LANDSAT full scenes) it is often sufficient to run the transformation on a smaller, representative spatial subset in order to determine a

Table 6

Variance-covariance matrix of the MAD variates for the Jülich images from June 26 and August 292001 after one application of the MAD transformation

\begin{tabular}{llllll}
\hline 1.54 & 0.00 & 0.00 & 0.00 & 0.00 & 0.00 \\
0.00 & 1.25 & 0.00 & 0.00 & 0.00 & 0.00 \\
0.00 & 0.00 & 0.89 & 0.00 & 0.00 & 0.00 \\
0.00 & 0.00 & 0.00 & 0.61 & 0.00 & 0.00 \\
0.00 & 0.00 & 0.00 & 0.00 & 0.52 & 0.00 \\
0.00 & 0.00 & 0.00 & 0.00 & 0.00 & 0.30 \\
\hline
\end{tabular}

Table 7

As Table 6 after iteration to convergence

\begin{tabular}{lrrrrr}
\hline 5.85 & 3.87 & -1.53 & 0.62 & -2.49 & -3.19 \\
3.87 & 9.17 & -3.61 & -0.01 & -2.70 & -3.26 \\
-1.53 & -3.61 & 5.51 & -0.22 & 1.40 & 1.08 \\
0.62 & -0.01 & -0.22 & 1.93 & -0.73 & -1.00 \\
-2.49 & -2.70 & 1.40 & -0.73 & 2.40 & 2.53 \\
-3.19 & -3.26 & 1.08 & -1.00 & 2.53 & 3.50 \\
\hline
\end{tabular}


Table 8

Weighted variance-covariance matrix of the MAD variates after iteration to convergence

\begin{tabular}{llllll}
\hline 0.664 & 0.000 & 0.000 & 0.000 & 0.000 & 0.000 \\
0.000 & 0.507 & 0.000 & 0.000 & 0.000 & 0.000 \\
0.000 & 0.000 & 0.295 & 0.000 & 0.000 & 0.000 \\
0.000 & 0.000 & 0.000 & 0.122 & 0.000 & 0.000 \\
0.000 & 0.000 & 0.000 & 0.000 & 0.043 & 0.000 \\
0.000 & 0.000 & 0.000 & 0.000 & 0.000 & 0.021 \\
\hline
\end{tabular}

satisfactory regression for radiometric normalization (Schroeder et al., 2006). For mosaicing applications, only overlapping areas can be used, again corresponding to relatively small array dimensions. The iterated MAD scheme thus provides a fast, reliable and robust method for automatic radiometric normalization of multispectral image time series in most situations that one might expect to encounter in practice.

\section{Acknowledgment}

The work presented here was carried out partly within the Global Monitoring for Security and Stability (GMOSS) Network of Excellence of the European Commission.

\section{References}

Abrams, M., Hook, S., \& Ramachandran, B. (1999). ASTER user handbook. Technical report. Jet Propulsion Laboratory and California Institute of Technology.

Aiazzi, B., Alparone, L., Baronti, S., \& Garzelli, A. (2002). Context-driven fusion of high spatial and spectral resolution images based on oversampled multiresolution analysis. IEEE Transactions on Geoscience and Remote Sensing, 40(10), 2300-2312.

Canty, M. J. (2007). Image analysis, classification, and change detection in remote sensing, with algorithms for ENVI/IDL. Taylor and Francis.

Canty, M. J., \& Nielsen, A. A. (2006). Visualization and unsupervised classification of changes in multispectral satellite imagery. International Journal of Remote Sensing, 27(18), 3961-3975. Internet http://www.imm. dtu.dk/pubdb/p.php?3389

Canty, M. J., Nielsen, A. A., \& Schmidt, M. (2004). Automatic radiometric normalization of multitemporal satellite imagery. Remote Sensing of Environment, 91(3-4), 441-451. Internet http://www.imm.dtu.dk/pubdb/p. php?2815

Coppin, P., Jonckheere, I., Nackaerts, K., \& Muys, B. (2004). Digital change detection methods in ecosystem monitoring: A review. International Journal of Remote Sensing, 25(9), 1565-1596.
Du, Y., Teillet, P. M., \& Cihlar, J. (2002). Radiometric normalization of multitemporal high resolution images with quality control for land cover change detection. Remote Sensing of Environment, 82, 123-134.

Furby, S. L., \& Campbell, N. A. (2001). Calibrating images from different dates to like-valuecounts. Remote Sensing of Environment, 77, 186-196.

Hall, F. G., Strebel, D. E., Nickeson, J. E., \& Goetz, S. J. (1991). Radiometric rectification: toward a common radiometric response among multidate, multisensor images. Remote Sensing of Environment, 35, 11-27.

Hotelling, H. (1936). Relations between two sets of variates. Biometrika, 28, 321-377.

Li, H., Manjunath, B. S., \& Mitra, S. K. (1995). A contour-based approach to multisensor image registration. IEEE Transactions on Image Processing, 4(3), 320-334.

Moran, M. S., Jackson, R. D., Slater, P. N., \& Teillet, P. M. (1992). Evaluation of simplified procedures for retrieval of land surface reflectance factors from satellite sensor output. Remote Sensing of Environment, 41, 160-184.

Nielsen, A. A. (2007). The regularized iteratively reweighted MAD method for change detection in multi- and hyperspectral data. IEEE Transactions on Image Processing, 16(2), 463-478. Internet http://www.imm.dtu.dk/pubdb/ p.php?4695

Nielsen, A. A., Conradsen, K., \& Simpson, J. J. (1998). Multivariate alteration detection (MAD) and MAF post-processing in multispectral, bitemporal image data: New approaches to change detection studies. Remote Sensing of Environment, 64, 1-19. Internet http://www.imm.dtu.dk/pubdb/p.php?1220

Radke, R. J., Andra, S., Al-Kofahi, O., \& Roysam, B. (2005). Image change detection algorithms: A systematic survey. IEEE Transactions on Image Processing, 14(4), 294-307.

Redner, R. A., \& Walker, H. F. (1984). Mixture densities, maximum likelihood and the EM algorithm. SIAM Review, 26(2), 195-239.

Schmidt, M., King, E., \& McVicar, T. R. (2005). Towards an internally consistent calibration for 11 AVHRR instruments in a 20-year time series of satellite data. In W. P. Menzel \& T. Iwasaki (Eds.), Proc. 4th International Asia-Pacific Remote Sensing Symposium, Honolulu (pp. 5658). Bellingham, Wash.. SPIE

Schott, J. R., Salvaggio, C., \& Volchok, W. J. (1988). Radiometric scene normalization using pseudo-invariant features. Remote Sensing of Environment, 26, 1-16.

Schroeder, T. A., Cohen, W. B., Song, C., Canty, M. J., \& Zhiqiang, Y. (2006). Radiometric calibration of Landsat data for characterization of early successional forest patterns in western Oregon. Remote Sensing of Environment, 103(1), 16-26.

Singh, A. (1989). Digital change detection techniques using remotely-sensed data. International Journal of Remote Sensing, 10(6), 989-1002.

Wiemker, R. (1997). An iterative spectral-spatial Bayesian labeling approach for unsupervised robust change detection on remotely sensed multispectral imagery. Proceedings of the 7th International Conference on Computer Analysis of Images and Patterns, vol. 1296 (pp. 263-370). LCNS.

Yang, X., \& Lo, C. P. (2000). Relative radiometric normalization performance for change detection from multi-date satellite images. Photogrammetric Engineering and Remote Sensing, 66, 967-980. 